\title{
Knowledge Sharing on the Semantic Web
}

\author{
Nicholas J. Kings ${ }^{1}$, Caroline Gale ${ }^{2}$, and John Davies ${ }^{1}$ \\ ${ }^{1}$ Next Generation Web Research Group, BT Group, Adastral Park, Ipswich, UK \\ \{nick.kings, john.nj.davies\}@bt.com \\ ${ }^{2}$ Chimera - Institute for SocioTechnical Research and Innovation, Essex University, UK \\ cgaledessex.ac.uk
}

\begin{abstract}
This paper details the design, implementation and evaluation of an ontology-based knowledge sharing tool. The system, "Squidz", automatically classifies browsed web pages against an ontology, and allows users to share comments made about those pages to members of a community. As the user browses web pages, recommendations of relevant documents which have already been shared are produced, based upon both the user's social network as well as the semantic content of the page currently in view. Key to the design of the system has been the requirement, evidenced by earlier studies, that sharing should be easily effected as a side-effect of browsing rather than comprising a separate and distracting task. Another feature of the system is the linkage of a formal ontology with user-provided tags of shared information, thus combining the proven popularity of folksonomy-based systems with the shared and formal domain model provided by an ontology.
\end{abstract}

Keywords: Semantic Web, Knowledge Sharing, Tagging, Social Software, Communities of Practice.

\section{Introduction}

In general, knowledge sharing tools combine the functions of searching for and distributing information. As a user requires information to undertake a task, information relevant to that task can be located. Underpinning knowledge sharing tools is the premise that someone in the user's wider community has already created or accessed relevant information (explicit knowledge transfer) or someone is able to provide help or advice (tacit knowledge transfer).

Knowledge management can be defined as the "systematic application of actions to ensure that an organisation obtains greatest benefit from the information that is available to it" [1]. Knowledge sharing software supports the activities of collating, categorising and distributing information [2], which creates a group memory and improves team awareness [3, 4].

The application of a knowledge sharing tool has a direct impact on the community's behaviour; any interactive digital technology has embedded implicit cultural assumptions. Raybourn et al. [5] suggest that there is no recipe or standard format for encouraging participation, nor should any one cultural perspective be forced on such a community. However, functions to support the community may be just as important 
as other functions supported by a system [5-10]. Kings et al. [11, 12] further suggest that a sense of history and a user's reputation are prerequisites for the development of a shared community purpose.

\subsection{Models of Knowledge Sharing}

The Semantic Web [13] can provide enhanced information access based on the exploitation of machine-processable meta data. Central to the vision of the Semantic Web are ontologies, which are seen as facilitating knowledge sharing and re-use between agents, be they human or artificial [14]. They offer this capability by providing a consensual and formal conceptualisation of a given domain. As such, the use of ontologies and supporting tools offer an opportunity to significantly improve knowledge management capabilities on the intranets of organisations and on the wider web. Furthermore, Mika [15] suggests that although the Semantic Web has been defined to facilitate machine understanding of the World Wide Web, the process of creating and maintaining that shared ontology is a purely social activity. Each ontology is created in a process that requires a group, or community, to build and share an agreed understanding of the important concepts and objects for that self same community. Mika further proposes that the understanding of social presence is crucial in understanding how an ontology evolves and gains acceptance.

Recently, we have seen the emergence of a number of very popular communitybased systems on the Web. Typically in systems such as flickr ${ }^{1}$ and delicious ${ }^{2}$, instead of using a centralized form of classification, users are encouraged to assign freely chosen keywords, called tags, to pieces of information or data, a process known as tagging. As a community of users generate a series of tags for overlapping and common items, a "folksonomy" can been seen to emerge. Since folksonomies are incremental and end user-generated and therefore inexpensive to implement, advocates of folksonomy believe that it provides a useful low-cost alternative to more traditional controlled vocabularies or classification schemes.

The combination of social networking systems such as $\mathrm{FOAF}^{3}$ and $\mathrm{XFN}^{4}$ with the development of tag-based folksonomies, implemented as blogs and wikis initally were seen as a basis for the semantic environment. This has been seen as part of a change from accessing static web pages to the use of the web as an application platform [16]:

- The change from centralised information sources to an approach of creating and distributing Web content itself, characterised by open communication, the willingness to share and re-use information, and "the market as a conversation" [17].

- The change from using web sites as point sources of information, stored within static Web pages, to sources of remotely accessible information, through the use of network accessible APIs or services.

In contrast to defined, formal taxonomies, categories in a folksonomy may appear to be arbitrary and idiosyncratic. However, a particular tag is chosen for a particular Web page based upon an individual's own understanding of the content being tagged,

\footnotetext{
${ }^{1}$ http://www.flickr.com/

${ }^{2} \mathrm{http}: / /$ del.icio.us/

${ }^{3} \mathrm{http}: / / \mathrm{xmlns.com} /$ foaf/0.1/

${ }^{4}$ http://gmpg.org/xfn/
} 
which combines the personal, social, and technical understanding of that content [15]. By publishing a tag and tagged content to a wider audience, other users are subtly encouraged to explore other tagged content and other users' interests.

Pind [18] suggests that there is anecdotal evidence to show that the sets of tags used within a community tend to converge upon a common set of agreed meanings and usage. Pind further suggests, however, that tagging software could be improved by the addition of the following five features: the software should suggest appropriate tags; the software should display related tags and topics; suggest tags that others have used to describe the same items; infer topic hierarchies from the way tags are used; and, allow a user to quickly edit and change tags that have already been applied. The Squidz tool addresses all of these issues.

In Section 2, we describe the Squidz tool. Two key features of the system are, firstly, the requirement, as evidenced by earlier studies, that sharing should be easily effected as a side-effect of browsing rather than comprising a separate and distracting task [19-21]; and, secondly, the linkage of a formal ontology with user-provided tags of shared information, thus combining the proven popularity of folksonomy-based systems with the shared and formal domain model provided by an ontology. Section 3 describes the operation of the software in more detail. Section 4 details the evaluation of Squidz.

\section{Software Overview}

The main function of Squidz is to share knowledge in the form of textual annotations about web pages, within a community of business users. The secondary purpose of Squidz is to allow a user to discover new social contacts or sources of information, through discovery of shared interests. The important features to be implemented by the software are:

- To improve software adoption, Squidz must be useful to an individual user without relying on any other's contributions. Squidz should be able to be used as an advanced book marking tool.

- All users can view and comment upon any other's annotations.

- An annotation is made in a technical and a social context. The technical context is represented by the topics associated with the annotation; the social context is represented by the community where the annotation is posted.

- The software filters and presents appropriate annotations, based upon each user's technical and social context. In effect, a relevant annotation is one made by a closely related person within a topic area related to the current page.

- Squidz models a user's social network in simple manner [2]: sharing pages to oneself; sharing to close work or team colleagues; sharing to members of a community of practise; or, sharing to all users of the system

Squidz is implemented as a browser plug-in, making use of a number of web services to classify browsed web pages and to retrieve annotations made by other members of the user community; Squidz is just one potential interface to the meta-data generated within the community. Squidz is being developed for communities of interest, or practice, within a corporate environment. In common with other tools being 
developed within the $\mathrm{SEKT}^{5}$ project, Squidz is utilising the PROTON ontology [22, 23]; PROTON ${ }^{6}$ is an ontology for knowledge management, modelling entities such as Documents with associated Topics as well as Users with Interests and Communities of Users.

As a user browses the internet or their intranet, the web page is classified against the formal ontology, and that classification is used to retrieve related pages. By clicking on the icons and words in the plug-in, separate information windows are created, rather than attempting to merge information within the browsed page.

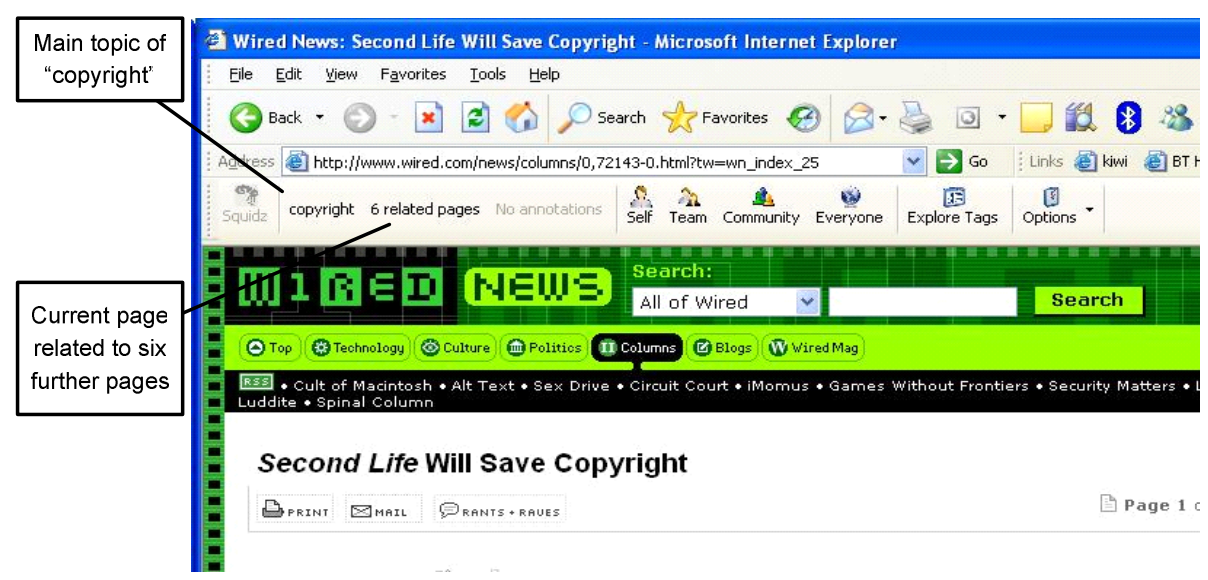

Fig. 1. Pages classified during browsing

Figure 1 shows the main user interface to Squidz, after a user has been browsing: web page's main topic is "copyright" and that six related annotated pages have been previously shared by other users; the main topic for a web page is either the highest ranked topic returned by the classifier or, if this user has already annotated this page, the main topic is taken from that annotation.

\subsection{System Architecture}

Squidz has been implemented as a number of server side components and the user interface is supplied as an Internet Explorer Browser Helper Object (or plug-in). The server side components write to a database which is mapped to an OWL ontology (PROTON) held in KAON. Information generated by using Squidz is thus fully accessible to other semantic web applications. Thus, other applications developed within SEKT, such as Semantic Search and Browse, are enable to share information through a semantic repository [22, 24-26]. Squidz has been envisaged as being used with a corporate setting, for use within communities of practise or communities of interest. Squidz monitors and tracks a user's browsing behaviour; privacy and secrecy were seen as less relevant within an enterprise setting. For use of Squidz within a

\footnotetext{
${ }^{5} \mathrm{http} / / /$ www.sekt-project.com/

${ }^{6} \mathrm{http} / / /$ proton.semanticweb.org/
} 
community of users across the wider internet, further work would be required to satisfy concerns about user security and privacy.

For use within British Telecom (BT), BT procures the Inspec and ABI bibliographic record databases, giving access to over 4 million bibliographic records. The format for each bibliographic record is based on ISO 2709 (which is based on the Library of Congress MARC format ${ }^{7}$ ).

A classifier has been developed, which assigns subject categories from the ABI and Inspec controlled vocabularies to content retrieved from the Web [27]. The classifier identifies the occurrence of controlled indexing terms in the text, and selects those terms for classification that are deemed most significant. The significance of each controlled indexing term is not only dependent on its frequency of occurrence in the text, but also on its inter-dependencies with other controlled indexing terms. The classifier web service returns the four highest ranking subject topics, for each web page, as well as up to ten of the most significant words and phrases identified in the text.

An inspection of the classifications presented by the classifier for a number of web pages suggests that it is capable of producing sensible classifications for previously unseen Web content. However, a full evaluation of the classification technique is currently being undertaken.

\subsection{Semantic Annotations}

When sharing a web page in Squidz, the user has the opportunity to provide some folksonomy-style tags which describe the content of the page, along with a comment about the page. Squidz models an annotation as the following: the user's comment; a set of formal topics, generated by the page classifier; a set of informal user-supplied tags; the target audience (individual, team, community or world as chosen by the user); and, a set of keywords and phrases, also generated by the page classifier. For each user tag, Squidz derives an associated set of keywords; each tag is semantically characterised by that keyword vector rather that the character string the user chose to represent the concept denoted by that particular tag.

As web pages are annotated with tags, the set of words for each tag is re-calculated automatically based upon the keywords stored within the page annotation. The keywords are associated with each tag in order to find related pages even if pages have been tagged with different terms; the derived keywords allow pages to be tagged with similar words, even though each user has their own understanding of that word. For example, one user's tag of "project" may represent the same concept as another user's tag of "SEKT". Adding an annotation forces the system to recalculate the keywords associated with the user's tags and subsequently all pages that are now related to the current page.

Though a folksonomy allows a community to evolve their own vocabulary, at a low cost for each contributor, there are associated problems of synonomy and polysemy [28], as there is no central defined meaning for each keyword or phrase used. Using the approach proposed, the semantic annotation uses the information available from the tagged pages themselves to provide a common basis for understanding the community's concepts.

\footnotetext{
${ }^{7}$ http://www.loc.gov/marc/
} 
Annotations are also made to a particular context, such as "Self", "Team", "Community" or "Everyone". This is used as a recommendation as to whom the annotation may be most relevant; by taking into account the suitability of an annotation, this may reduce the "cost" of a user understanding the importance or relevance of that particular web page [2].

As pages are shared in Squidz, a number of OWL metadata elements are generated and stored in the PROTON ontology: a Squidz annotation is represented as a PROTON document with an associated set of ontology topics and informal usergenerated tags. Thus, the community generates relevant annotated content for its own use and this content can be accessed via other Semantic Web applications, such as the Semantic Search and Browse tool in BT's Digital Library [22, 26].

\section{Squidz in Action}

Section 2 presented an overview of Squidz, whereas this section examines each of the main software functions in more detail.

\subsection{Fetching Related Pages}

Squidz provides the user with "peripheral vision" of previously shared web pages and annotations related to the current page. As a web page is viewed by a user, a request for related pages and their associated annotations is made to the remote web service. Related pages are determined in the following manner:

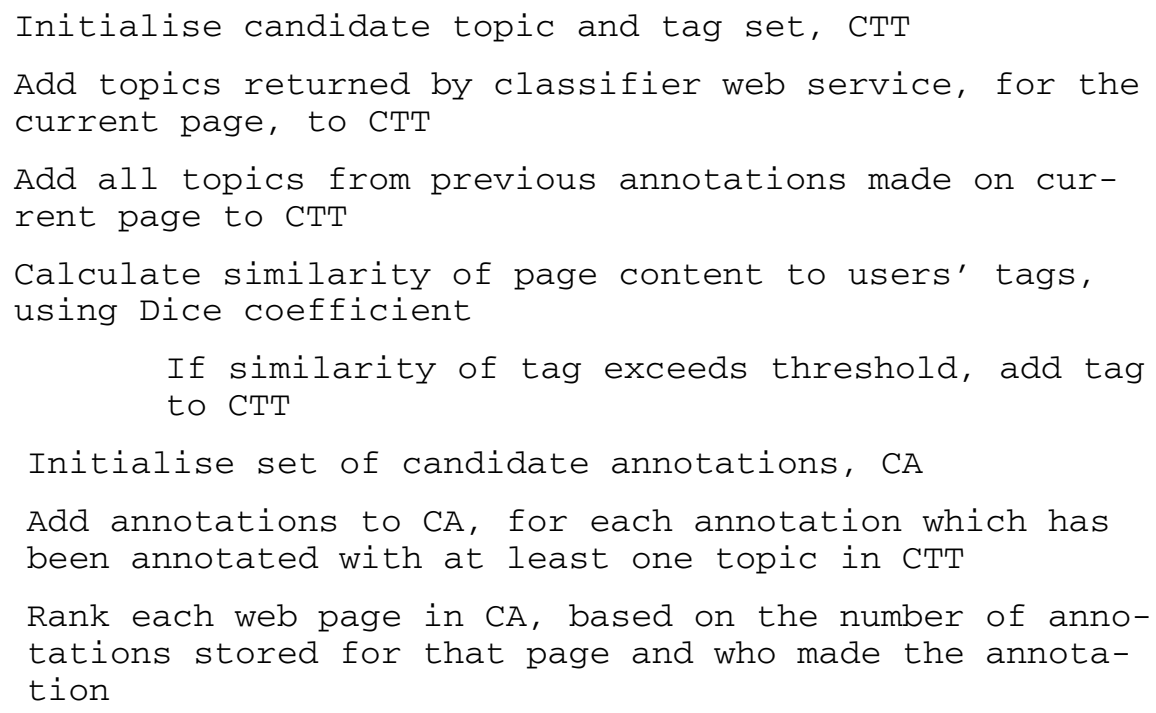

The similarity of each tag in the system to the current page content is calculated with the Dice coefficient, as shown in Equation 1 [29], where KWC and KWT are 
sets of keywords: KWC, the set of keywords derived for a viewed page; and, KWT, the set of keyword associated with a particular user tag.

$$
\operatorname{Dice}(K W C, K W T)=\frac{|K W C \cap K W T|}{\frac{1}{2}(|K W C|+|K W T|)}
$$

The similarity measure is taken between the currently viewed page's content and a tag's keyword vector rather than between the current page contents and the keywords associated with each previously stored page for two reasons. Firstly, this decision improves the performance: as there are fewer user tags than annotations, fewer similarity calculations need to be under taken. Secondly, by matching user tags, Squidz is returning pages that are associated with a user's concepts: one user may be interested another user's "project" pages, without actually knowing of that other person's interest.

In effect, each annotation "votes" for a web page to be brought to the user's attention; the weight associated to the vote varies based on who made the annotation. Thus, there is a chance that annotations made by people outside of a user's direct social network will be brought to a user's attention. In this manner, the technical or topical context of web page and well as a user's social context is taken into account to account to rank a related page.

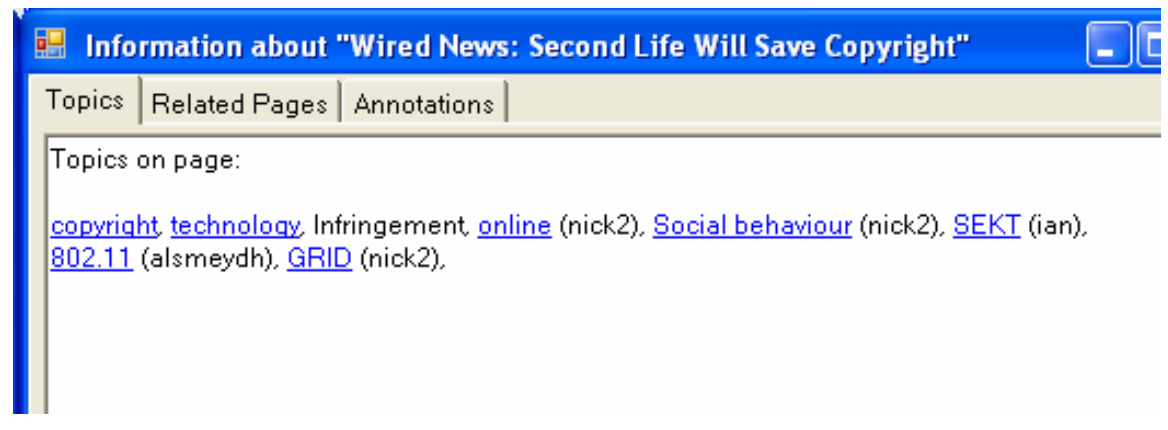

Fig. 2. Topics related to current page

Figure 2 shows that eight topics have been identified that are related to the current page: three topics from the defined topic ontology, and, five user created tags: user- generated tags are identified by placing the name of the user who generated the tag in parentheses. As described above, the defined topics have been identified by the classifier.

The list of related user tags, shown in Figure 2, is formed from the tags found within the returned list of related pages. Each underlined topic or tag can be clicked to cause all of the annotations made with that particular topic to be retrieved and displayed. Related topics are calculated in the following manner:

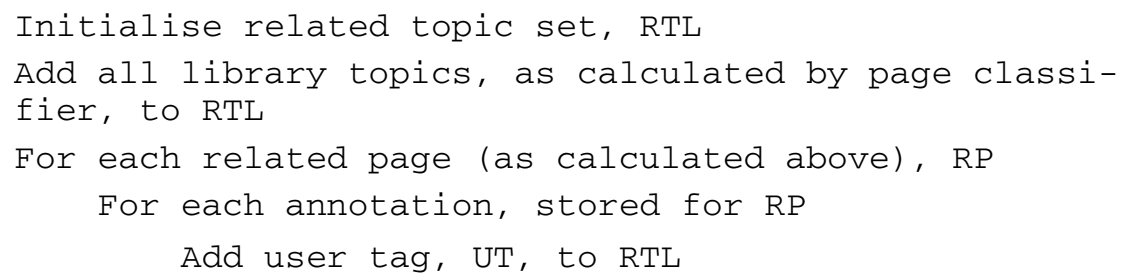


Thus, the list of related topics contains ontology topics, user tags that are similar to the current page and user tags that have been explicitly added to those related pages. Only if an annotation has been created with a particular topic or tag, will the name be clickable; Figure 2 shows no annotations have been made with the topic of "Infringement".

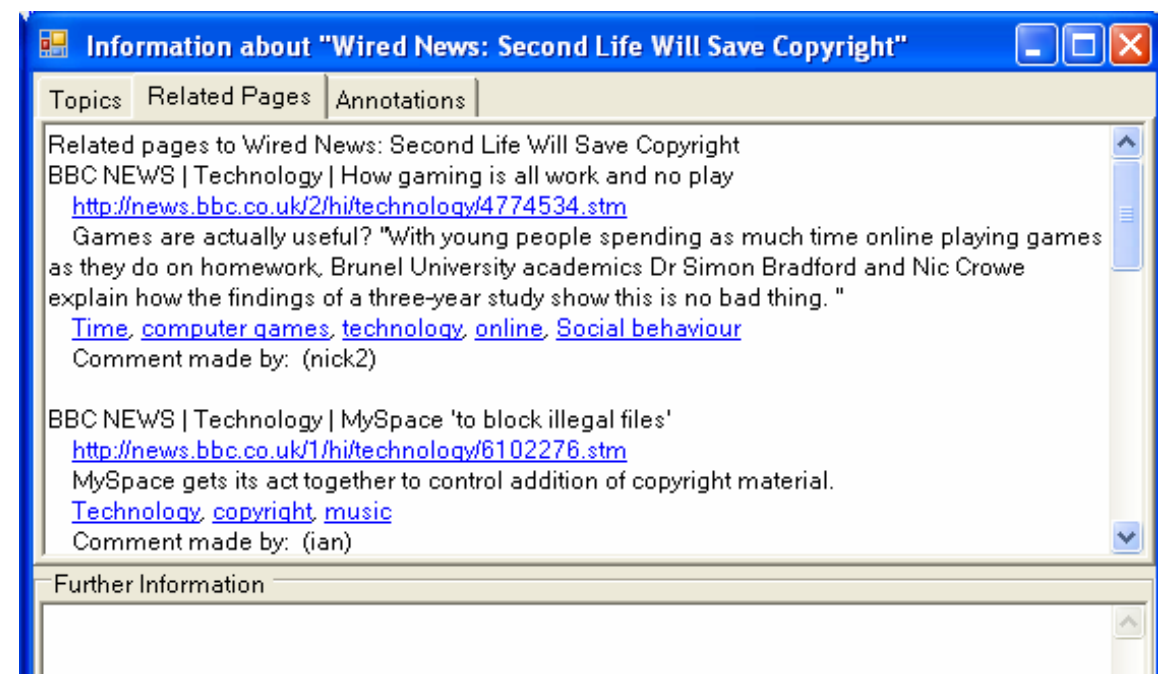

Fig. 3. Pages related to current page, ranked by social network

Figure 3 shows the scrollable list of annotated pages found to be related to the currently viewed web page. As in Figure 2, clicking an underlined topic will retrieve annotations; clicking an underlined URL will cause the browser to load that particular page. Implementing the user interface in this manner allows a user to explore across web pages, rather than having to visit a particular website and then start exploring tags and relations from that point onwards.

\subsection{Sharing Annotations}

By clicking on "Self", "Team", "Community" or "Everyone" (as shown Figure 1), a user can choose to share an annotation about the currently viewed web page. This is implemented via a less intrusive pop-up window, rather than by changing the contents of the main browser widow. Figure 4 shows that comments about a particular web page about to be shared to members of this user's community.

Sharing to a particular set of people does not preclude that others outside of group will not see the annotation, as having the ability to view every person's contribution is a crucial way of building an active set of users. However, the user-chosen target group of the annotation is used in the ranking algorithm when calculating related pages: a page shared to "Team" by one of my team members is ranked higher than a page shared by the same person but shared to "Community". 


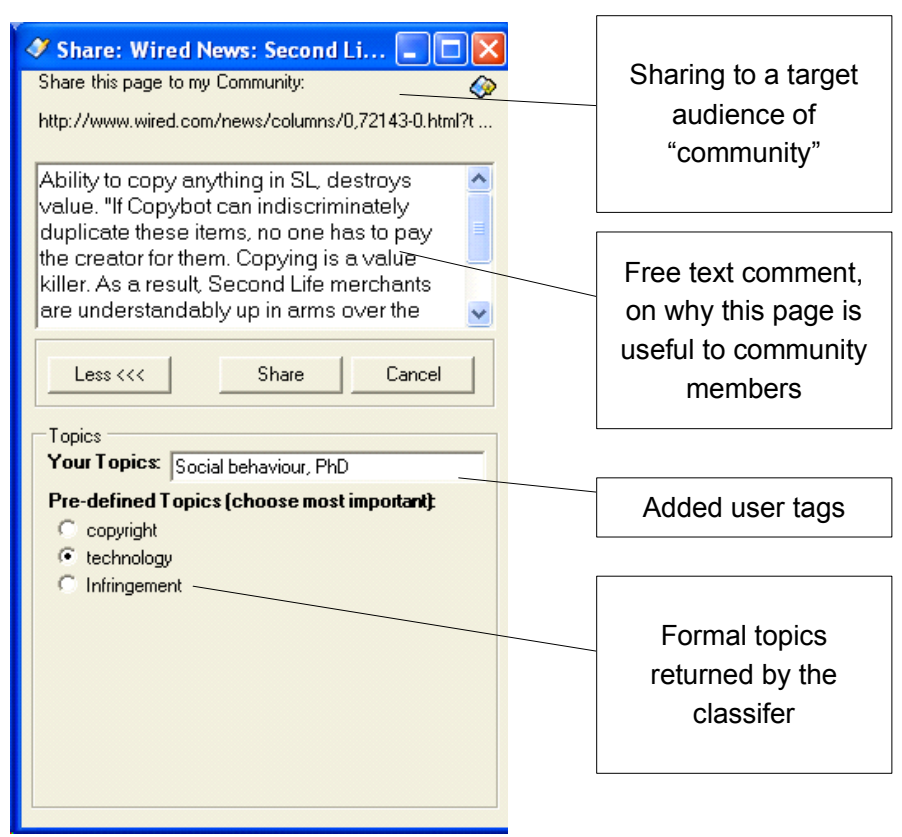

Fig. 4. Storing an annotation with the user choosing to add two tags and changing the main topic

Figure 5 shows that after the annotation has been made, the web page is now related to nine pages, and the main formal topic for the page is shown as "technology".

By making an annotation the topics related to this page have changed, as shown by comparing Figures 2 and 6. As the added annotation explicitly mentions the user's tag of "PhD", this tag would be expected to be shown for this page. However, by annotating, the algorithm has calculated that there is also a similarity between this page and the user's tags of "Tagging" and "Classification techniques". Figure 6 also shows that "Infringement" can now be clicked, as there has been an annotation stored for that topic.

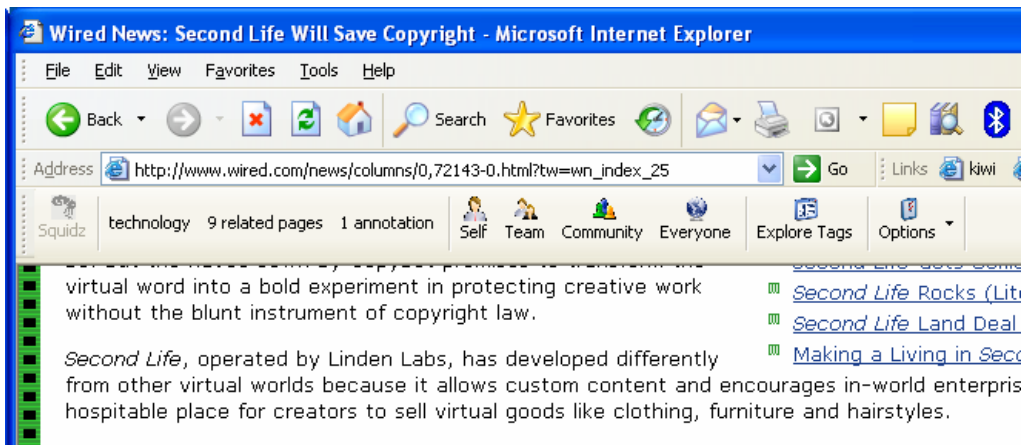

Fig. 5. Further related pages after adding an annotation 
期 Information about "Wired News: Second Life Will Save Copyright" 口回匈 Topics |Related Pages | Annotations |

Topics on page:

copyriaht, technology Infringement online (nick2), Social behaviour (nick2), SEKT (ian), PhD (nick2), Taqqing (nick2), 802.11 (alsmeydh), Classification techniques (nick2), GRID (nick2),

Fig. 6. Recalculated related topics, after the annotation has been added

\subsection{Exploring Annotations}

Squidz can be used as a personal "book marking" tool as well as for information sharing. Because of the problems that information sharing systems require a critical mass of users [4], one of the requirements for Squidz was that the software should be useful for a single user. However, in order to support longer term community spirit, it is important that all annotations should be visible to anyone using Squidz. By developing Squidz in this manner, information sharing to the community is then a by-product of a simple, well understood action of "book marking" a web page.

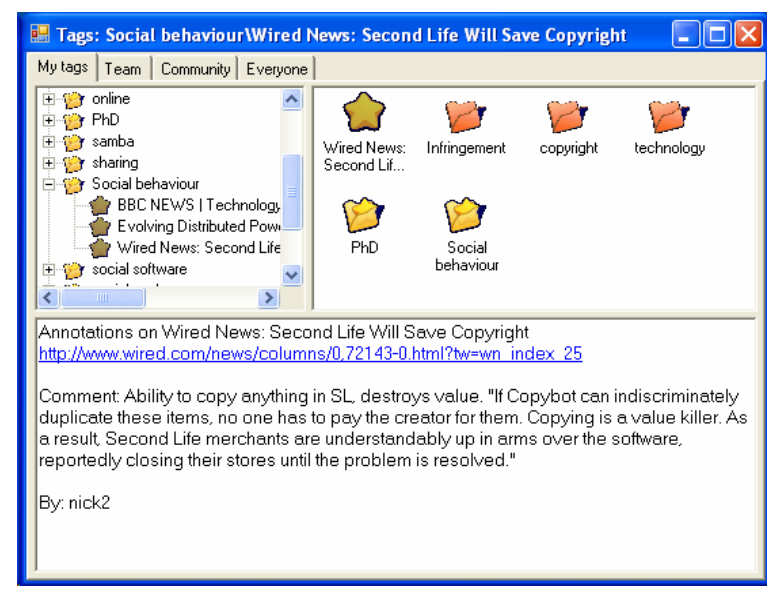

Fig. 7. Exploring my tags

Figures 7 and 8 show a user exploring the topics and tags to find web pages. The user interface allows the annotations to be separated into annotations made by your self, your team members, your community members and everyone. Figure 9 shows the annotation made on a particular web page: the web page can be found by either exploring the user's tags or exploring the formal topic hierarchy; the web page can be found through multiple routes, as it is associated with a number of different topics. A user' tags are displayed as yellow folders, and their own annotations are shown as yellow stars, while formal topics are shown as red folders. 


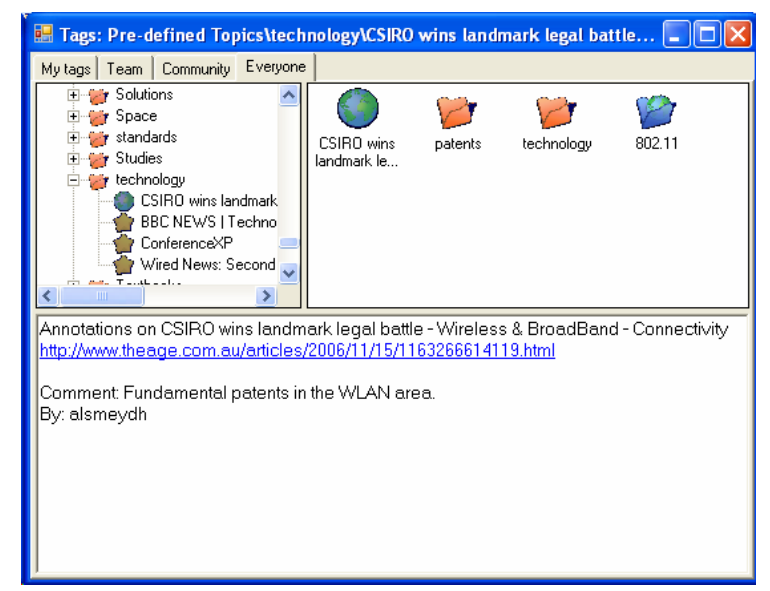

Fig. 8. Exploring another user's annotations and tags

Figure 8 shows a user exploring all the annotations made by everyone. Here annotations made by other people are denoted by blue globes, and their tags are denoted by blue folders. Figure 8 also shows that four annotations have been made with the topic of "technology": three have been by made by the user himself, and a fourth has been made by user "alsmeydh". From this screen, it can be seen that there is a user tag of "802.11", and that tag can be subsequently explored.

\section{Evaluation}

\subsection{Related Work}

The design for Squidz has been influenced by lessons learnt from the Jasper [30] and OntoShare [20, 21] knowledge sharing tools. In those tools, a profile of user interests is built to filter information, based upon which web pages have been shared by various users. Notifications, about pages being stored, were sent by email which caused, however this caused a disconnect between sharing and commenting and there was little dialogue encouraged between users. Sharing information should be easily effected as a side-effect of browsing rather than comprising a separate and distracting task.

Piggy Bank [31] presents a tool integrated into a web browser, coupled with the use of associated RDF collection utilities. Like Squidz, Piggy Bank allows a community of users to share and collaborate over items of information found. The approach taken by Piggy Bank, however, is to present the RDF metadata directly in the user interface; Squidz simplifies the user interface and only exposes parts of the metadata associated with document sharing, such as topic and user.

Annotea [32, 33] is a framework to support collaboration and sharing of semantically marked objects. The toolset allows users to tag Web pages with concepts, and allow the metadata to be repurposed through various XSLT style sheets. Annotea provides a flexible interface to explore bookmarks and topics. Squidz, however, is aimed 
at providing page recommendations, through the classification of the current web page and retrieval of related items.

Onomi [34] is also a social bookmarking tool for use within a corporate environment. Onomi also builds a semantic description of the users' tags, however, the technique used within Onomi relies on stemming user tags to provide the common basis for understanding, rather than the more sophisticated use of keyword sets, as used within Squidz.

\subsection{Experimental Design}

Squidz has been being subjected to a three-stage user-centred evaluation. For the first stage, a heuristic evaluation [35] of the user interface was undertaken. A small group of five researchers, acting as usability experts, judged whether the user interface adhered to a list of established usability heuristics; a checklist was adapted from the Xerox heuristic evaluation system checklist [36]. A number observations were made, most of which were concerned with minor interface problems and system performance. The results of the evaluation were collated and discussed with the development team. Squidz was then modified in accordance with these observations.

The second stage comprised a cognitive walkthrough evaluation [37]. Users were asked to use Squidz in order to complete a number of knowledge sharing tasks, where the user's actions and behaviours will be recorded. Users were encouraged to talk through their actions and their concerns as they undertake each task, as this provided additional information about the usability of Squidz and the user's thought processes as they used the application. At the end of each task, users were also asked to complete a short questionnaire. The findings were again discussed with the Squidz development team. By the completion of this stage, all major interface issues had been resolved.

At the time of submitting this paper, the third and final stage of evaluation is underway: preliminary results are given in the next section. Squidz is being rolled out for use within an intranet, to users with a range of technical experience. This stage consists of a series of semi-structured interviews to find out how useful people have found Squidz [38]. The purpose of the interviews is to validate the following hypotheses: knowledge sharing requires both a technical and social context; sharing is more likely to occur if the costs of sharing are reduced; and, sharing allows the knowledge of the community to built and enhanced.

\subsection{Results and Analysis}

Currently, twenty users have registered and downloaded the software: nine of those are regular users and a further eight users have made at least one annotation. All of the users are familiar with information sharing with tools such as email and delicious. Longer term user acceptance will be an important aspect to measure, since Squidz constantly monitors web page access. As the current trial has only been running a relatively short time at the time of writing, and further longitudinal data is required to determine the users' long term attitude to sharing information in this manner.

Nevertheless, data from the current valuation has validated the Squidz approach: users have commented on the simplicity and ease of use for the tool. The user 
interviews have also identified a number of new functions that could be incorporated into later versions of the software. For example, one requested function would be ability to "notify immediately" other people that a page has been annotated, as well still having the ability to be made aware of that page while browsing.

The users found the Squidz ranking of pages taking into account the content of the page and the original sharer of the page natural and intuitive. Annotations were typically tagged with only a few keywords or phrases. The characterization of tags with a keyword vector, however, gives a richer semantic representation of tags than in typical user-tagging applications. Semantic tags allowed users to name topics closer to the user's own higher level concepts, rather than each user having to add explicitly the complete set of keywords describing each web page.

\section{Discussion and Conclusions}

In this paper we have presented the design, implementation of a semantic tool for annotating and sharing information about web pages. Squidz is intended to test the hypothesis that information sharing is more effective when the software is aware of both the social and technical context of that information. The approach taken is to associate a formal topic ontology alongside an informal folksonomy, through the ability to annotate web pages. Presenting information on related pages and topics, through the toolbar, allows a user to browse and explore when convenient to the user, rather than forcing a particular mode of usage; knowledge sharing occurs as a result of normal user activity (browsing). Though further improvements to the tool are planned, Squidz has already gained positive user feedback and acceptance.

Acknowledgements. The work described in this paper was developed as part of the SEKT project, under EU funding (IST IP 2003506826). Further project details may be found at http://www.sekt-project.com/.

\section{References}

1. Marwick, A.D., Knowledge management technology. IBM Systems Journal, (2001). 40(4): p. 814-830.

2. Shneiderman, B., Leonardo's Laptop: Human Needs and the New Computing Technologies. (2003), London, England: MIT Press.

3. Udel, J., The New Social Enterprise. InfoWorld, (2004). Vol. 26(No. 13): p. 47,50-52.

4. Rafaeli, S. and D.R. Raban, Information sharing online: a research challenge. International Journal of Knowledge and Learning, (2005). 1(2): p. 62-79.

5. Raybourn, E.M., N. Kings, and J. Davies, Adding cultural signposts in adaptive community-based virtual environments. Interacting with Computers, (2003). 15(1): p. 91-107.

6. Preece, J., Online Communities: Designing Usability, Supporting Sociability. (2000), Chichester, England: John Wiley \& Sons.

7. Merali, Y. and J. Davies. Knowledge capture and utilization in virtual communities. in International Conference On Knowledge Capture. (2001). Victoria, British Columbia, Canada.

8. Kim, A.J., Community Building on the Web. (2000), Berkeley, Ca: Peachpit Press. 
9. Crossley, M., N.J. Kings, and J.R. Scott, Profiles - Analysis and Behaviour, in Location and Personalisation: Delivering Online and Mobility Services, D. Ralph and S. Searby, Editors. (2004), IEE: London.

10. Sharratt, M. and A. Usoro, Understanding Knowledge-Sharing in Online Communities of Practice. Electronic Journal of Knowledge Management, (2003). 1(2): p. 187-196.

11. Kings, N. Knowledge sharing using Semantic Web technologies. in 1st Workshop on Friend of a Friend, Social Networking and the Semantic Web. (2004). Galway, Ireland.

12. Kings, N., D. Alsmeyer, and F. Owston, Libraries as Shared Spaces, in Universal Access in HCI: Inclusive Design in the Information Society. Proceedings of HCI International, 2003, Volume 4. (2003), Lawrence Erlbaum Associates.

13. Berners-Lee, T., J. Hendler, and O. Lassila, The Semantic Web. Scientific American, (2001). May, 2001.

14. Fensel, D., Ontologies: Silver Bullet for Knowledge Management and Electronic Commerce. (2001): Springer-Verlag, Berlin.

15. Mika, P. Ontologies Are Us: A Unified Model of Social Networks and Semantics. in The Semantic Web - ISWC 2005. (2005). Galway, Ireland: Springer.

16. O'Rielly, T., What Is Web 2.0: Design Patterns and Business Models for the Next Generation of Software. (2005), O'Reilly Media, Inc.

17. Levine, R., et al., The Cluetrain Manefesto: The End of Business as Usual. (2000), London: Pearson Education.

18. Pind, L., Folksonomies: How we can improve the tags. (2005), Pinds.com.

19. Davies, J., S. Stewart, and R. Weeks. Knowledge Sharing over WWW. in WebNet '98. (1988).

20. Davies, J., A. Duke, and A. Stonkus, OntoShare: Evolving Ontologies in a Knowledge Sharing System, in Towards The Semantic Web, J. Davies, D. Fensel, and F.v. Harmelen, Editors. (2003), John Wiley \& Sons, Ltd.: Chichester. p. 161-177.

21. Davies, J., A. Duke, and Y. Sure, OntoShare: a knowledge management environment for virtual communities of practice, in Proceedings of the international conference on Knowledge capture. (2003), ACM Press: Sanibel Island, FL, USA.

22. Bontcheva, K., et al., Semantic Information Access, in Semantic Web Technologies: Trends and Research in Ontology-based Systems, J. Davies, R. Studer, and P. Warren, Editors. (2006), John Wiley \& Sons Ltd: Chichester, England.

23. Kirakov, A., Ontologies for Knowledge Management, in Semantic Web Technologies: Trends and Research in Ontology-based Systems, J. Davies, R. Studer, and P. Warren, Editors. (2006), John Wiley \& Sons Ltd: Chichester, England.

24. Davies, J., et al., Next generation knowledge management. BT Technology Journal, (2005). 23(3): p. 175-190.

25. Glover, T. and J. Davies, Integrating device independence and user profiles on the Web. BT Technology Journal, (2005). 23(1): p. 239-248.

26. Warren, P., I. Thurlow, and D. Alsmeyer, Applying Sematic Technology to a Digital Library, in Semantic Web Technologies: Trends and Research in Ontology-based Systems, J. Davies, R. Studer, and P. Warren, Editors. (2006), John Wiley \& Sons Ltd: Chichester, England.

27. Thurlow, I. Classifying Web content for a corporate digital library. in London Communications Symposium. (2006).

28. Golder, S. and B.A. Huberman, Usage Patterns of Collaborative Tagging Systems. Journal of Information Science, (2006). Vol. 32(No. 2): p. pp198-208.

29. Grossman, D.A. and O. Frieder, Information Retrieval: Algorithms and Heuristics, ed. T.I.R. Series. (2006): Springer. 
30. Davies, J., S. Stewart, and R. Weeks, Knowledge Sharing Agents over the WWW. BT Technology Journal, (1998). Vol. 16(No. 3, July).

31. Huynh, D., S. Mazzocchi, and D. Karger. Piggy Bank: Experience the Semantic Web Inside Your Web Browser. in International Semantic Web Conference 2005. (2005). Galway, Ireland: Springer.

32. Koivunen, M.-R. Annotea and Semantic Web Supported Collaboration. in ESWC 2005, UserSWeb workshop. (2005).

33. Koivunen, M.-R. Semantic Authoring By Tagging with Annotea Social Bookmarks and Topics. in SAAW2006 - 1st Semantic Authoring and Annotation Workshop. (2006). Athens, GA, USA.

34. Damianos, L., J. Griffith, and D. Cuomo. Onomi: Social Booking on a Corporate Intranet. in Collaborative Web Tagging Workshop. (2006). WWW2006, Edinburgh, UK.

35. Neilsen, J. and R. Molich, Heuristic Evaluation of User Interfaces, in Proceedings of the SIGCHI conference on Human factors in computing systems. (1992): Monterey, California, United States. p. $373-380$.

36. Christiansson, P., Heuristic Evaluation - A System Checklist. (2000), Xerox Corporation.

37. Wharton, C., et al., Applying Cognitive Walkthroughs to more Complex User Interfaces: Experiences, Issues, and Recommendations, in Proceedings of the SIGCHI conference on Human factors in computing systems. (1992): Monterey, California, United States. p. 381-388.

38. Reynolds, T.J. and J. Gutman, LADDERING THEORY, METHOD, ANALYSIS, AND INTERPRETATION. Journal of Advertising Research, (1988)(February/March). 